\title{
REMINERALIZATION EFFECT OF EGGSHELL POWDER ON POST-ORTHODONTIC WHITE SPOT LESIONS COMPARED TO CPP-ACP: A RANDOMIZED CONTROLLED CLINICAL TRIAL
}

\author{
Mohammad A. Sarhan*, Maha A. El Baz** and Omaima S. Hamza***
}

\begin{abstract}
Aim: White spot lesions are the only carious process lesions that can be remineralized noninvasively. The focus of modern dentistry is stopping the caries process without unnecessary damaging the tooth structure. The present clinical trial aimed to assess the remineralizing effect of eggshell powder (ESP) compared to Casein Phosphopeptide-Amorphous Calcium Phosphate (CPP-ACP) in carious post-orthodontic white spot lesions orthodontic treatment.
\end{abstract}

Methodology: Twenty-Four patients were selected from the Clinic of Orthodontic Dentistry Department, Faculty of Dentistry, Cairo University. Patients were divided into two groups according to the treatment received either ESP gel or CPP-ACP paste (GC Tooth-Mousse). The participants were instructed to use pea size eggshell powder gel or GC Tooth Mousse on the teeth surfaces using a clean finger twice daily after brushing their teeth with Colgate Total Original Care toothpaste. WSLs were assessed at baseline, 1 month, 3months and 6 months by DIAGNOdent (Kavo, Germany).

Results: Data were analyzed using Two-way ANOVA showed that there was no statistically significant difference in overall DIAGNOdent mean readings between ESP and GC Tooth Mousse groups at $(\mathrm{P}=0.136)$.

Conclusion: Based on the data obtained in this study, it is suggested that ESP is effective and has a promising future in the treatment of post-orthodontic WSLs.

KEY WORDS: White spot lesion, initial caries lesions, remineralization.

* MSc Degree Candidate, Conservative Dentistry Department, Faculty of Dentistry, Cairo University ** Associate Professor, Conservative Dentistry Department, Faculty of Dentistry, Cairo University

*** Professor, Conservative Dentistry Department, Faculty of Dentistry, Cairo University 


\section{INTRODUCTION}

Dental caries is a noteworthy public health problem, which has a high prevalence worldwide. It is a complex, multifactorial disease involving many risk and protective factors. It is a biofilm dependent disease process whereby the bacterial acid and products diffuse and demineralize enamel and dentin. The process of caries is well known as a pattern of alternating demineralization and remineralization cycles of tooth minerals Young et al $(\mathbf{2 0 1 5})^{(1)}$.

The caries process includes a wide range of events from early molecular changes in the apatite crystals of the tooth, to a visible white spot lesion (WSL), followed by dentin involvement and eventual cavitation. Progression through these stages requires a persistent imbalance between risk and protective factors that result in the dissolution of apatite crystals and the net loss of calcium $(\mathrm{Ca})$, fluoride $(\mathrm{F})$, phosphate $(\mathrm{PO} 4)^{-3}$ and other ions from the dental hard tissues Featherstone $(\mathbf{2 0 0 8})^{(2)}$.

WSL is the earliest stage of enamel caries that can be detected by the naked eye. It appears as chalky white opacity as a result of subsurface demineralization of enamel by bacterial organic acids. The demineralization process causes increased porosity in the enamel structure which modifies its color and glossiness by the increase of light scattering optical property of the decalcified enamel. Thus, the enamel loses its normal translucency and white opaque lesions appear Deveci et al. (2018) ${ }^{(3)}$.

WSLs are prevalent in patients with bonded orthodontic appliances. The presence of braces, bands, wires, and other devices of fixed orthodontic appliances increase plaque retention sites. Also, it makes the performance of proper oral hygiene by these patients more difficult, and the conditions are deteriorated by expanding the duration of the orthodontic treatment. This requires the need for special precautions and steps to be taken to counteract the development of these carious lesions Sundararaj et al. $(2015)^{(4)}$.
In contrast to cavitated carious lesions, WSL can be reversed by re-establishing a balance between demineralization and remineralization. Several remineralizing agents have demonstrated a positive effect on WSL including natural product-based agent CPP-ACP, which is based on milk products Bailey et al. (2009) ${ }^{(5)}$, grape seed extracts Benjamin et al. (2012) ${ }^{(6)}$, ginger, honey, and rosemary Gocmen et al. $(\mathbf{2 0 1 6})^{(7)}$. This research was conducted to compare the remineralization efficacy of eggshell powder gel and CPP-ACP (GC Tooth-Mousse).

Eggshell powder gel high calcium content has the potential to favor the remineralization process for initial caries lesions. Also, eggshell is considered inexpensive, abundant and safe compared to current materials used for this purpose. The null hypothesis tested in the current study was that there is no difference between CPP-ACP and eggshell powder gel in the treatment of white spot lesions.

\section{MATERIALS AND METHODS}

This is a single centered, single blinded (assessor), two parallel armed randomized control clinical trial. The study was carried out on 24 participants who had just finished orthodontic treatment in the orthodontic dentistry department in The Faculty of Dentistry, Cairo University, Egypt. 24 participants were divided into two groups: 12 for control (CPP-ACP) and other: 12 for intervention (Eggshell powder gel). Simple randomization was done by generating numbers from 1:24 using Sequence Generator, Randomness and Integrity Services Ltd (https://www.random.org/). Opaque sealed envelopes containing the generated numbers were used to insure allocation concealment. This study was approved by Research Ethics Committee of Faculty of Dentistry, Cairo University (CREC), approval no. 18- 6-19.

\section{Eligibility criteria}

The participants were enrolled in this study in accordance of the following inclusion and exclusion criteria. 


\section{Inclusion criteria of the participants}

- Patient who has just completed a comprehensive orthodontic treatment.

- Presence of at least one WSL, DIAGNOdent score $(0-20)$.

- Age range between 13 and 35years.

\section{Exclusion criteria of the participants}

- Patients unable to return for recall appointments.

- High caries risk.

- Have plaque accumulation and a periodontal problem.

- In progress treatment of a chronic disease.

- Presence of abnormal oral, medical, or mental condition (including any milk-related allergies or any medical condition involving kidneys or salivary glands).

- Presence of dentin caries, enamel hypoplasia or restorations on teeth of interest

\section{Clinical procedure}

The change in the lesion fluorescence was evaluated using diode laser fluorescence device (DIAGNOdent). The device was calibrated before each surface was analyzed. Based on the previous study by Yazıcıoğlu and Ulukapı (2014) $)^{(8)}$, laser fluorescence examination results had been scored using the manufacturer's scoring system.

\section{Clinical evaluation of WSLs}

After drying with gauze, the tip of DIAGNOdent device was positioned on the buccal surface, performing exploratory movements until the highest value (peak) was obtained. Three measurements were taken and averaged to give the final test value.

The labial surface of each tooth in both the jaws from central incisor to the first molar was considered in this study. Scores were taken at the baseline, 1, 3 and 6 months after the first application of the products, which continued through the whole follow up period. The primary endpoint was the change in fluorescence of the lesions after 6 months.

\section{Sample preparation and application of reminer- alzaing agents}

\section{Nano-eggshell powder preparation}

Based on a previous study Elsalamony et al. (2018) $^{(9)}$, organic chicken eggs were obtained from a supermarket were cleaned thoroughly using distilled water to remove any dirt. A small crack was made in each eggshell to split the shell into two halves to evacuate the egg content. The eggshells then were disinfected in a hot water bath at $100^{\circ} \mathrm{C}$ for 10 minutes. This step facilitated the detachment of the inner membrane manually.

Afterwards, the eggshell flakes were exposed to steam sterilization to ensure that it is pathogen free. Then the eggshells were crushed using a sterile

TABLE (1): Criteria used in examination with DIAGNOdent Yazıcığlu and Ulukapı (2014), laser fluorescence score recommended by manufacturer.

\begin{tabular}{|l|l|}
\hline Score & Description \\
\hline Score 1 & Laser fluorescence score 0-4, no caries, or white opaque lesions. \\
\hline Score 2 & Laser fluorescence score 5-10, enamel caries limited to the outer half of the enamel thickness. \\
\hline Score 3 & $\begin{array}{l}\text { Laser fluorescence score 11-20, enamel caries limited to the inner half of the enamel thickness without obvious spread } \\
\text { in the dentin. }\end{array}$ \\
\hline Score 4 & Laser fluorescence score $\geq 21$, caries spread in the dentin. \\
\hline
\end{tabular}


mortar and pestle to prepare them for machine milling. The crushed particles then were milled using a zirconia ball-milling machine (Planetary Ball Mill Fritsch Pulverisette) at 200 rpm for 24 hours. This procedure resulted in a nano-sized eggshell powder, which facilitated its incorporation in to a gel form.

\section{Preparation of eggshell hydrogel formulations}

Eggshell powder hydrogel dispersions were prepared through dispersing a suitable concentration of egg shell powder in distilled water, followed by dissolving the gelling agent; sodium carboxymethyl cellulose at a concentration of $5-10 \%$ under continuous stirring at $1000 \mathrm{rpm}$ using a magnetic stirrer. The formed hydrogel was kept at $8^{\circ} \mathrm{C}$ (in the refrigerator) for a period of 24 hours before further use. $10 \%$ concentrations of eggshell powder produced a paste of consistency that is appropriate for handling and application.

\section{Eggshell powder gel application}

Participants were supplied by the main researcher with ESP gel and $1450 \mathrm{ppm}$ fluoride toothpaste (Colgate Total Original Care toothpaste). They were instructed to use pea size eggshell powder gel on the tooth surfaces using a clean finger twice daily (after breakfast and before bedtime) after brushing their teeth with Colgate Total Original Care toothpaste. The participants were told to keep the eggshell gel over the teeth surfaces for a minimum of $3 \mathrm{~min}$ before rinsing. After the application of eggshell gel, the participants should not drink or eat anything for at least $30 \mathrm{~min}$. No other fluoride supplements (toothpaste, mouthwash and fluoride tablets) were allowed during the trial.

\section{GC Tooth Mousse (CPP-ACP) application Karabekiroğlu et al. (2017) ${ }^{(10)}$}

Participants were supplied by the main researcher with CPP-ACP crème (GC Tooth Mousse) and 1450 ppm fluoride toothpaste (Colgate Total Original Care toothpaste). They were instructed to use pea size GC Tooth Mousse on the tooth surfaces using a clean finger twice daily (after breakfast and before bedtime) after brushing their teeth with Colgate Total Original Care toothpaste). The participants were told to keep the CPP-ACP crème over the teeth surfaces for a minimum of $3 \mathrm{~min}$ before rinsing. After the application of CPP-ACP, the participants should not drink or eat anything for at least 30 min. No other fluoride supplements (toothpaste, mouthwash and fluoride tablets) were allowed for use.

\section{Statistical analysis}

Statistical analysis was performed using IBM SPSS Statistics Version 2.0 for Windows. Data was presented as mean, standard deviation (SD), frequencies (n) and percentages. KolmogorovSmirnov and Shapiro-Wilk tests were used to assess data normality of continuous data. The significance level was set at $P \leq 0.05$. Two-way ANOVA was conducted to investigate the effect of study variables and their interaction on remineralization of white spot lesions. Mann-Whitney test was performed for intergroup comparisons and Kruskal-Wallis test and Wilcoxon signed-rank test were used for multiple comparisons. Chi-square test was used to compare frequency distribution of categorical data.

\section{RESULTS}

1. Two-way ANOVA for the effect of remineralizing agent and evaluation time on remineralization of white spot lesions

Two-way ANOVA (table 2) showed that "evaluation time" had a significant effect on remineralization of white spot lesions $(\mathrm{P}<0.001)$. While "remineralizing agent" had no significant effect on remineralization of white spot lesions $(\mathrm{P}=0.917)$. The interaction between "remineralizing agent x evaluation time" also did not have a significant effect on remineralization of white spot lesions $(\mathrm{P}=0.372)$. 
TABLE (2): Two-way ANOVA for the effect of study variables and their interaction on remineralization of white spot lesions.

\begin{tabular}{|l|c|c|c|c|c|}
\hline & Type III sum of squares & df & Mean square & F & Sig. \\
\hline Remineralizing agent & 0.224 & 1 & 0.224 & 0.011 & $0.917 \mathrm{NS}$ \\
\hline Evaluation time & 609.344 & 3 & 203.115 & 9.815 & $<0.001 *$ \\
\hline Remineralizing agent $x$ Evaluation time & 65.021 & 3 & 21.674 & 1.047 & $0.372 \mathrm{NS}$ \\
\hline
\end{tabular}

*: significant at $P \leq 0.05 ;$ NS: non-significant at $P>0.05$

2. Overall DIAGNOdent readings within each remineralizing agent and evaluation time group

Univariate analysis of variance showed that there was no statistically significant difference in overall DIAGNOdent mean readings between eggshell powder and GC Tooth Mousse groups at $\mathrm{P}=0.917$ (table 3). On the other hand, there was a statistically significant difference in overall DIAGNOdent mean readings between evaluation time groups at $\mathrm{P}<0.001$ (table 4). Baseline conceded the statistically significantly highest overall readings, followed by 1 month and 3 months which were statistically similar. While 6 months showed the statistically significantly lowest overall readings.

TABLE (3): Mean \pm SD and P-value for the comparison of overall DIAGNOdent readings between remineralizing agent groups.

\begin{tabular}{|c|c|c|}
\hline Eggshell powder & GC Tooth Mousse & P-value \\
\hline $9.94 \pm 5.02$ & $9.93 \pm 4.44$ & $0.917 \mathrm{NS}$ \\
\hline
\end{tabular}

NS: non-significant at $P>0.05$

TABLE (4): Mean \pm SD and P-value for the comparison of overall DIAGNOdent readings between evaluation time groups.

\begin{tabular}{|l|l|l|l|l|}
\hline Baseline & $\mathbf{1}$ month & $\mathbf{3}$ months & 6 months & P-value \\
\hline $11.87 \pm 4.58^{\mathrm{a}}$ & $10.32 \pm 4.46^{\mathrm{b}}$ & $9.50 \pm 4.55^{\mathrm{b}}$ & $7.78 \pm 4.57^{\mathrm{c}}$ & $<0.001^{*}$ \\
\hline
\end{tabular}

*: significant at $P \leq 0.05$

Means with different superscript letters are statistically significantly different at $P \leq 0.05$
3. Effect of different evaluation times on remineralization of white spot lesions within each remineralizing agent

Kruskal-Wallis test followed by Wilcoxon signed-rank test (table 5) showed that within eggshell powder group, there was a statistically significant difference in DIAGNOdent readings at $\mathrm{P}<0.001$. Six months follow up displayed the statistically significantly lowest readings, but did not differ significantly from that of 3 months. The readings at baseline, 1 month and 3 months did not show any significant difference. While within GC Tooth Mousse group, there was no statistically significant difference in DIAGNOdent readings between different evaluation times at $\mathrm{P}=0.102$.

TABLE (5): Mean \pm SD and P-value for the comparison of DIAGNOdent readings within each remineralizing agent at different evaluation times.

\begin{tabular}{|c|c|c|}
\hline & Eggshell powder & GC Tooth Mousse \\
\hline Baseline & $12.14 \pm 4.45^{\mathrm{a}}$ & $11.55 \pm 4.77^{\mathrm{a}}$ \\
\hline $\mathbf{1}^{\text {month }}$ & $10.78 \pm 4.82^{\mathrm{a}}$ & $9.77 \pm 3.99^{\mathrm{a}}$ \\
\hline 3 months & $9.32 \pm 5.00^{\mathrm{ab}}$ & $9.68 \pm 4.10^{\mathrm{a}}$ \\
\hline 6 months & $7.11 \pm 4.54^{\mathrm{b}}$ & $8.56 \pm 4.55^{\mathrm{a}}$ \\
\hline P-value & $<0.001^{*}$ & $0.102 \mathrm{NS}$ \\
\hline
\end{tabular}

*: significant at $P \leq 0.05$

Means with different superscript letters within each column are statistically significantly different at $P \leq 0.05$ 
4. Effect of remineralizing agent on remineralization of white spot lesions at each evaluation time

Mann-Whitney test (table 6) showed that there were no statistically significant differences in readings between eggshell powder and GC Tooth Mousse groups at baseline $(\mathrm{P}=0.502), 1$ month $(\mathrm{P}=0.471), 3$ months $(\mathrm{P}=0.459)$ and 6 months $(\mathrm{P}=0.136)$.

TABLE (6): Mean \pm SD and P-value for the comparison of DIAGNOdent readings between remineralizing agents at each evaluation time.

\begin{tabular}{|c|c|c|c|c|}
\hline & Baseline & $\mathbf{1}$ month & $\mathbf{3}$ months & 6 months \\
\hline $\begin{array}{c}\text { Eggshell } \\
\text { powder }\end{array}$ & $12.14 \pm 4.45$ & $10.78 \pm 4.82$ & $9.32 \pm 5.00$ & $7.11 \pm 4.54$ \\
\hline $\begin{array}{c}\text { GC Tooth } \\
\text { Mousse }\end{array}$ & $11.55 \pm 4.77$ & $9.77 \pm 3.99$ & $9.68 \pm 4.10$ & $8.56 \pm 4.55$ \\
\hline P-value & $0.502 \mathrm{NS}$ & $0.471 \mathrm{NS}$ & $0.459 \mathrm{NS}$ & $0.136 \mathrm{NS}$ \\
\hline
\end{tabular}

*: significant at $P \leq 0.05 ;$ NS: non-significant at $P>0.05$

\section{DISCUSSION}

WSL is an initial carious lesion which is confined to the enamel, and is the only stage of caries process that can be reversed by remineralization. The focus of modern dentistry is to intervene non-invasively and to conservatively remineralize enamel without unnecessary damaging the tooth structure. The aim of this study was to evaluate the remineralization potential of ESP compared to CPP-ACP on postorthodontic WSLs. Sundararaj et al. (2015) ${ }^{(4)}$ showed significant high prevalence of WSLs in patients during their orthodontic treatment.

Laser-induced fluorescence is a spectroscopic method in which a molecule is excited by the absorption of laser light followed by spontaneous emission of light at a wavelength longer than the excitation wavelength. Previous investigation revealed that dental caries exhibit readily detectable fluorescence unlike sound dental tissues. Fluorescent properties of dental caries can be attributed to an increase in bacterial flora and its metabolism, mainly bacterial endogenous porphyrins (protoporphyrin IX (PPIX), meso-porphyrin, and copro-porphyrin) Shakibaie et al. (2011) $)^{(11)}$.

DIAGNODent (KaVo, Germany) is a portable diode laser device, which emits light of wavelength $655 \mathrm{~nm}$ (red end of the electromagnetic spectrum) and measures the fluorescence quantitatively. Light from a laser diode is coupled into an optical fibre and transmitted to the tooth. The excitation fibre is surrounded by a bundle of 9 thin concentric fibres, which gather fluorescence as well as backscattered light and guide it to the detection unit, where the intensity of the fluorescent radiation is presented as a digital value (on a 0-99 scale) Diniz et al. (2015) $^{(12)}$.

Several studies had shown that DIAGNOdent can be applied in dental practice for quantification of WSLs. Aljehani et al. (2006) ${ }^{(13)}$ showed that DIAGNOdent is appropriate for the detection and monitoring of post-orthodontic WSLs. Fluorescence values of post-orthodontic WSLs is a sign of endogenous porphyrins and other molecules of bacterial origin, which present in the porous enamel caries lesions. While the remineralization of postorthodontic WSLs, as seen in the present study, can be attributed to a reduction of the cariogenic and microbial activity, rather than mineral gain, which is indicated by lower DIAGNOdent score.

Nascimento et al. (2017) ${ }^{(14)}$ displayed in their systematic review that fluoride application can reduce the risks of WSLs in orthodontic patients, but not reducing the degree of pre-existing lesions. Longbottom et al. (2009) ${ }^{(15)}$ considered that calcium and phosphate remineralization technique is promising in adjunct to fluoride. Philip (2019) ${ }^{(16)}$ highlighted the need for developing new approaches to remineralize initial caries lesions. 
CPP-ACP is a nanocluster that binds calcium and phosphate ions in an amorphous form. During acid attacks it buffers the salivary and biofilm $\mathrm{pH}$ by releasing calcium and phosphate ions, thus preserving calcium and phosphate supersaturated in the saliva, which precipitates as calcium-phosphate compound onto the exposed tooth surface Gurunathan et al. (2012) ${ }^{(17)}$. The use of CPP-ACP, may be a helpful in preventing or remineralizing WSLs, but there is conflicting evidence of its efficacy. Mendes et al. (2018) $^{(18)}$ in a randomized clinical trial found that acidulated fluoride and $\mathrm{CPP}-\mathrm{ACP}$ crème separately had the same remineralization effect on WSLs when assessed using DIAGNOdent, while combining both agents, results were the best.

Bailey et al. (2009) ${ }^{(5)}$ displayed in their clinical trial that significantly more post-orthodontic WSLs regressed with the CPP-ACP cream compared with a placebo over 3 months. While Tao et al. (2018) (19) in a systematic review observed that fluoride combined with CPP-ACP achieved no clinical advantage over fluorides monotherapy using Visual inspection scores, laser fluorescence, and quantitative light-induced fluorescence assessments on smooth surface WSLs. The justification the author mentioned in this study was that smooth surfaces are less susceptible to caries, and fluoride alone might be enough to remineralize these lesions. Surprisingly, for occlusal initial caries lesions the results showed that the combination treatment of CPP-ACP and fluoride showed significantly better efficacy than fluorides monotherapy.

In addition, Pulido et. al (2008) ${ }^{(20)}$ found no significant differences between the effects of CPP$\mathrm{ACP}$ and artificial saliva on the reduction of caries progression in vitro. And they suggested to apply CPP-ACP products for longer period of time (3 min) as was instructed to the participants in our study. While, Yadav et al. (2019) ${ }^{(21)}$ also in an in vitro study assessed the aesthetic improvement and healing capacity of WSLs using bioactive glass
(BiominF), CPP-ACP paste with fluoride \& ICON resin infiltration. Their results showed that ICON improved the color significantly compared to other groups, while CPP-ACP improved the healing of WSLs at 6 months assessed with DIAGNOdent. This result is contrast with our study, where DIAGNOdent readings of CPP-ACP group didn't have any significant difference at any follow up.

Asokan et al. (2019)(22) in a systematic review studying non-fluoridated remineralizing agents found that CPP-ACP results showed significant reduction in initial caries lesions measures using the visual examination method. But the author found lack of evidence supporting any other nonfluoridated remineralizing agents. Karabekiroğlu et al. (2017) ${ }^{(10)}$ in a randomized clinical trial found that the daily application of CPP-ACP for 3 years was not better than normal oral hygiene measures in improving the WSLs appearance. However, in a recent systematic review, Ma et al. (2019) $)^{(23)}$ CPP-ACP is an excellent remineralizing agent, as it lowered enamel surface roughness, and recoverd the enamel surface microhardness of WSLs when compared to no treatment or placebo.

Llena et al. (2015) $)^{(24)}$ in a clinical trial found that CPP-ACFP DIAGNOdent score of WSLs significantly improved compared to CPP-ACP and fluoride varnish. These results were explained by the rapid formation of subsurface fluorapatite when CPP-ACFP was used. Imani et al. (2019) (25) in a systematic review showed that both CPPACP and CPP-ACFP are effective in decreasing the prevalence and treating WSLs during and after orthodontic treatment.

On the other hand, there is a continuous need for more natural sources of calcium and phosphate, that would be cost effective and economical. As a result, previous investigations were performed to find out a way to take an advantage of recycling the eggshells, as it is considered inexpensive, abundant and safe compared to current materials used for this purpose. 
Many researches had been done to evaluate its use as a human dietary oral calcium supplement and for the treatment of osteoporosis Imani et al. (2019) ${ }^{(25)}$.

However, only few studies were performed to evaluate its potential as a remineralizing agent. Haghgoo et al. (2016) ${ }^{(26)}$ showed that ESP significantly increased the microhardness of artificial enamel carious lesions. Feroz et al. (2017) (27) showed that ESP significantly reduced surface roughness of erosive enamel lesions. Mony et al. (2015) ${ }^{(28)}$ found that ESP solution can remineralize the enamel, and highlighted the need of studying another suitable vehicle for ESP.

In the current study, the remineralization effect of ESP gel was compared with CPP-ACP (GC tooth mousse). The gel consistency of the ESP facilitated its application to mimic that of the comparator. Elbahrawy and El-Dosoky (2019) ${ }^{(29)}$ compared the remineralization capability of artificially induced enamel caries lesions between ESP solution and ESP slurry. They found that both mixtures significantly increased the microhardness of the enamel, but there was no significant difference between the solution and slurry groups.

In addition to the treatment of WSLs, Feroz et al. (2017) ${ }^{(27)}$ evaluated the effect of ESP on enamel erosive lesions invitro found that ESP reduced the surface roughness and was effective against erosive enamel lesions. Similarly, Yaberi and Haghgoo (2018) $^{(30)}$ performed an in-situ study to compare the effects of NHA and ESP on the microhardness of third molars enamel following soft drink exposure, and found that both that NHA and ESP have the capability to remineralize erosive lesions.

In this study, the results showed that there were no statistically significant differences in DIAGNOdent readings between ESP gel and GC Tooth Mousse groups at each evaluation time period. In addition, within ESP gel group, there was a statistically significant difference in DIAGNOdent readings. Six months follow up displayed the statistically significantly lowest readings. These results agree with Mony et al. (2015) ${ }^{(28)}$, Haghgoo et al in 2016 $^{(26)}$, Elbahrawy and El-Dosoky (2019) ${ }^{(29)}$, and Mohamed et al. (2020) ${ }^{(31)}$ that ESP is effective and is promising in remineralization of enamel caries lesions.

On the other hand, CPP-ACP group didn't show statistically significant difference in DIAGNOdent readings between different evaluation times, these finding agree with Pulido et. al (2008) ${ }^{(20)}$ and Tao et al. (2018) ${ }^{(19)}$ results. This might be due to the rapid clearance time of GC Tooth Mousse from the tooth surface. Pulido et. al (2008) ${ }^{(20)}$ suggested that the major part of CPP-ACP binds to bacteria instead of the tooth surface. In that case, the application of GC Tooth Mousse should be prior to tooth brushing. While Asokan et al. (2019)(22) in a systematic review showed that CPP-ACP significantly reduced initial caries lesions measurements by visual examination method. From our analysis, we conclude that the null hypothesis can be accepted, that there was no significant difference between CPP-ACP and eggshell powder gel in the treatment of white spot lesions.

\section{CONCLUSIONS}

Under the limitation of the present trial the following conclusion can be mentioned:

1. Eggshell powder (ESP) gel has a remineralization potential that could be promising in preventing further progress of WSLs.

2. Regular and prolonged application of tested remineralizing agents could ensure sustainable remineralization.

\section{RECOMMENDATIONS}

More clinical trials and measurement devices are needed that evaluate the remineralization potential of ESP. 


\section{REFERENCES}

1. Young, D. A., Nový, B. B., Zeller, G. G., Hale, R., Hart, T. C., Truelove, E. L., and Kuehne, J. (2015). The American Dental Association caries classification system for clinical practice: a report of the American Dental Association Council on Scientific Affairs. The Journal of the American Dental Association, 146(2), 79-86.

2. Featherstone J. D. (2008). Dental caries: a dynamic disease process. Australian dental journal, 53(3), 286-291.

3. Deveci, C., Çınar, Ç., \& Tirali, R. E. (2018). Management of White Spot Lesions. Dental Caries-Diagnosis, Prevention and Management. InTech, 129-65.

4. Sundararaj, D., Venkatachalapathy, S., Tandon, A., \& Pereira, A. (2015). Critical evaluation of incidence and prevalence of white spot lesions during fixed orthodontic appliance treatment: A meta-analysis. Journal of International Society of Preventive \& Community Dentistry, 5(6), 433-439.

5. Bailey, D. L., Adams, G. G., Tsao, C. E., Hyslop, A., Escobar, K., Manton, D. J., Reynolds, E. C., \& Morgan, M. V. (2009). Regression of post-orthodontic lesions by a remineralizing cream. Journal of dental research, 88(12), 1148-1153.

6. Benjamin, S., Sharma, R., Thomas, S. S., \& Nainan, M. T. (2012). Grape seed extract as a potential remineralizing agent: a comparative in vitro study. The journal of contemporary dental practice, 13(4), 425-430.

7. Gocmen, G. B., Yanikoglu, F., Tagtekin, D., Stookey, G. K., Schemehorn, B. R., \& Hayran, O. (2016). Effectiveness of some herbals on initial enamel caries lesion. Asian Pacific Journal of Tropical Biomedicine, 6(10), 846-850.

8. Yazıcıoğlu, O., \& Ulukapı, H. (2014). The investigation of non-invasive techniques for treating early approximal carious lesions: an in vivo study. International Dental Journal, 64(1), 1-11.

9. Elsalamony, N.A., M.Abdel-Hamid, D., \& Bakir, N.G. (2018). Bioactivity of aNanoEggshell-modified Calcium Hydroxide Dental Cement: An In-vitro Study.

10. Karabekiroğlu, S., Ünlü, N., Küçükyilmaz, E., Şener, S., Botsali, M. S., \& Malkoç, S. (2017). Treatment of post-orthodontic white spot lesions with CPP-ACP paste: A three year follow up study. Dental materials journal, 36(6), 791-797.

11. Shakibaie, F., George, R., \& Walsh, L. J. (2011). Applications of laser induced fluorescence in dentistry. International Journal of Dental Clinics, 3(3), 38-44.
12. Diniz, M. B., Campos, P. H., Sanabe, M. E., Duarte, D. A., Santos, M.T. B. R., Guaré, R. O., ...\& Rodrigues, J.A. (2015). Effectiveness of fluorescence-based methods in monitoring progression of noncavitated caries-like lesions on smooth surfaces. Operative dentistry, 40(6), E230-E241.

13. Aljehani, A., Yousif, M. A., Angmar-Månsson, B., \& Shi, X. Q. (2006). Longitudinal quantification of incipient carious lesions in postorthodontic patients using a fluorescence method. European journal of oral sciences, 114(5), 430-434.

14. Nascimento, P. L. D. M. M., Fernandes, M. T. G., Figueiredo, F. E. D. D., \& Faria-e-Silva, A. L. (2016). Fluoridereleasing materials to prevent white spot lesions around orthodontic brackets: a systematic review. Brazilian Dental Journal, 27(1), 101-107.

15. Longbottom, C., Ekstrand, K., Zero, D., \& Kambara, M. (2009). Novel preventive treatment options. In Detection, assessment, diagnosis and monitoring of caries (Vol. 21, pp. 156-163). Karger Publishers.

16. Philip N. (2019). State of the Art Enamel Remineralization Systems: The Next Frontier in Caries Management. Caries research, 53(3), 284-295.

17. Gurunathan, D., Somasundaram, S., \& Kumar, S. A. (2012). Casein phosphopeptide-amorphous calcium phosphate: a remineralizing agent of enamel. Australian dental journal, 57(4), 404-408.

18. Mendes, A. C., Restrepo, M., Bussaneli, D., \& Zuanon, A. C. (2018). Use of Casein Amorphous Calcium Phosphate (CPP-ACP) on White-spot Lesions: Randomised Clinical Trial. Oral health \& preventive dentistry, 16(1), 27-31.

19. Tao, S., Zhu, Y., Yuan, H., Tao, S., Cheng, Y., Li, J., \& He, L. (2018). Efficacy of fluorides and CPP-ACP vs fluorides monotherapy on early caries lesions: A systematic review and meta-analysis. PloS one, 13(4), e0196660.

20. Pulido, M. T., Wefel, J. S., Hernandez, M. M., Denehy, G. E., Guzman-Armstrong, S., Chalmers, J. M., \& Qian, F. (2008). The inhibitory effect of MI paste, fluoride and a combination of both on the progression of artificial carieslike lesions in enamel. Operative dentistry, 33(5), 550-555.

21. Yadav, P., Desai, H., Patel, K., Patel, N., \& Iyengar, S. (2019). A comparative quantitative \& qualitative assessment in orthodontic treatment of white spot lesion treated with 3 different commercially available materials - In vitro study. Journal of clinical and experimental dentistry, 11(9), e776-e782. 
22. Asokan, S., Geethapriya, P. R., \& Vijayasankari, V. (2019). Effect of nonfluoridated remineralizing agents on initial enamel carious lesions: A systematic review. Indian journal of dental research: official publication of Indian Society for Dental Research, 30(2), 282-290.

23. Ma, X., Lin, X., Zhong, T., \& Xie, F. (2019). Evaluation of the efficacy of casein phosphopeptide-amorphous calcium phosphate on remineralization of white spot lesions in vitro and clinical research: a systematic review and metaanalysis. BMC oral health, 19(1), 295.

24. Llena, C., Leyda, A. M., \& Forner, L. (2015). CPP-ACP and CPP-ACFP versus fluoride varnish in remineralisation of early caries lesions. A prospective study. European journal of paediatric dentistry, 16(3), 181-186.

25. Imani, M. M., Safaei, M., Afnaniesfandabad, A., Moradpoor, H., Sadeghi, M., Golshah, A., ... \& Mozaffari, H. R. (2019). Efficacy of CPP-ACP and CPP-ACPF for Prevention and Remineralization of White Spot Lesions in Orthodontic Patients: A Systematic Review of Randomized Controlled Clinical Trials. Acta Informatica Medica, 27(3), 199.

26. Haghgoo, R., Mehran, M., Ahmadvand, M., \& Ahmadvand, M. J. (2016). Remineralization effect of eggshell versus nano-hydroxyapatite on caries-like lesions in permanent teeth (in vitro). Journal of International Oral Health, 8(4), 435.
27. Feroz, S., Moeen, F., \& Haq, S. N. (2017). Protective effect of chicken egg shell powder solution (CESP) on artificially induced dental erosion: an in vitro atomic force microscope study. International Journal of Dental Sciences and Research, 5(3), 49-55.

28. Mony, B., Ebenezar, A. R., Ghani, M. F., \& Narayanan, A. (2015). Effect of chicken egg shell powder solution on early enamel carious lesions: an invitro preliminary study. Journal of clinical and diagnostic research: JCDR, 9(3), ZC30.

29. Elbahrawy, E. M. S., \& El-Dosoky, A. I. (2019). Remineralization Potential of Chicken Eggshell Powder in The Treatment of Artificially Induced Enamel Carious lesion in Permanent Teeth. Egyptian Dental Journal, 65(4-October (Fixed Prosthodontics, Dental Materials, Conservative Dentistry \& Endodontics)), 3581-3593.

30. Yaberi, M., \& Haghgoo, R. (2018). A comparative study of the effect of nanohydroxyapatite and eggshell on erosive lesions of the enamel of permanent teeth following soft drink exposure: A randomized clinical trial. Journal of International Oral Health, 10(4), 176.

31. Mohamed, E. O., Sharaf, A. A., Talaat, D. M., \& Nagui, D. A. (2020). Remineralization effect of egg shell powder and Novamine on initial caries-like lesions in young permanent teeth (in-vitro study). Alexandria Dental Journal. 